\title{
One-Pot Synthesis and Catalytic Properties of Encapsulated Silver Nanoparticles in Silica Nanocontainers
}

\author{
Magdalena Priebe and Katharina M. Fromm*
}

Core/shell and yolk/shell (nanorattles) nanoparticles (NPs) have been gaining an increasing interest due to the possibility to improve the properties of encapsulated material(s) by providing additional functions via the shell material(s). This can include beneficial modifications, such as enhancing stability and activity, controlling release and designing functionality. ${ }^{[1-3]}$ Therefore, those novel NPs exhibit a great potential in applications such as drug delivery, ${ }^{[1,2,4]}$ batteries, ${ }^{[1,2]}$ and catalysis. ${ }^{[1,2,5]}$ For example, a study of Yin et al. ${ }^{[6]}$ demonstrated that core/ shell $\mathrm{Au} @ \mathrm{Fe}_{2} \mathrm{O}_{3}$ deposited on $\mathrm{SiO}_{2}$ support performed better in catalytic oxidation of $\mathrm{CO}$ to $\mathrm{CO}_{2}$ than uncoated AuNPs on $\mathrm{SiO}_{2}$ support. Yao et al. ${ }^{[7]}$ tested metal nanoparticles (Fe, Co, Ni, $\mathrm{Ru})$ encapsulated in oxide shells $\left(\mathrm{SiO}_{2}, \mathrm{Al}_{2} \mathrm{O}, \mathrm{MgO}\right)$ in terms of catalytic conversion of $\mathrm{NH}_{3}$ into $\mathrm{H}_{2}$. Interestingly, the catalytic performance highly depended not only on the aforementioned presence of the shell but also on its composition. Silver nanoparticles (AgNPs) alone, core/shell Ag@SiO $2,{ }^{[8]}$ AgNPs supported on silica spheres, ${ }^{[9]}$ as well as AgNPs supported on silica ${ }^{[10]}$ were shown to serve as promising catalysts; however, to the best of the authors' knowledge, no study was done on catalytic properties of yolk/shell Ag@ $\mathrm{SiO}_{2}$.

While initial attempts to prepare hollow silica spheres have led to the formation of micron-sized particles with a broad size distribution, ${ }^{[11]}$ sol-gel reactions in water-in-oil microemulsion are now a commonly used procedure for the preparation of silica NPs with a narrow size distribution. ${ }^{[12,13]}$ Lin and co-workers ${ }^{[14]}$ reported the preparation of hollow silica NPs using a quaternary water-in-oil microemulsion consisting of cyclohexane/Triton X-100/n-hexanol/water. A reverse micelle acts here as a soft template where tetraethyl orthosilicate (TEOS) is initially hydrolyzed at the boundary phase and then silica is formed inside the micelle. Decreased condensation inside the silica sphere is provided by the use of a second silica precursor, 3-amino-propyltrimethoxy silane (APTS), and is due to an additional functional group, an amino group, making it less "condensable" compared with TEOS. Multiple washing processes lead then to a removal of unreacted silica precursors, and subsequently to the formation of hollow spheres. Interestingly, changing the reaction parameters, such as the sequence of the reagent addition or the TEOS/APTS ratio, solid spheres, yolk/shell spheres or hollow spheres of various sizes can be obtained. To overcome the problem of polydispersity, the same

M. Priebe, Prof. K. M. Fromm

Department of Chemistry

University of Fribourg

Chemin du Musée 9,

1700 Fribourg, Switzerland

E-mail: katharina.fromm@unifr.ch group reported that the composition of the micellar system can be changed from quaternary to ternary (cyclohexane/Igepal CO-520/water). ${ }^{[15]}$

Incorporation of other inorganic NPs into silica nanocontainers was so far achieved by two approaches: (a) addition of already synthesized NPs to the microemulsion ${ }^{[14]}$ or (b) introduction of a NP precursor into the micelles and reduction to the metal after the purification process. ${ }^{[15]}$ The former provides a mean for the preparation of superparamagnetic $\mathrm{Fe}_{3} \mathrm{O}_{4}$ inside fluorescent hollow silica nanocontainers, ${ }^{[14]}$ whereas the latter allows preparation of catalytic nanorattles with AuNPs inside hollow silica. ${ }^{[15]}$

Here, we demonstrate the preparation of AgNPs inside hollow $\mathrm{SiO}_{2}$ nanocontainers with catalytic properties. Our approach combines two of the aforementioned methods for the incorporation of AgNPs into $\mathrm{SiO}_{2}$ nanocontainers. Although $\mathrm{Ag} @ \mathrm{SiO}_{2}$ core/shell nanocomposites have been mentioned elsewhere, ${ }^{[8,16,17]}$ to the authors' best knowledge, it is the first time that nanorattles of Ag@hollow silica using one-pot reactions in microemulsion are reported and their catalytic properties are determined. The aim of this study is to present the best procedure for obtaining $\mathrm{Ag} @ \mathrm{SiO}_{2}$ nanorattles, and to also indicate how critical even slight changes in the experimental conditions can be on the final result.

Our procedure starts with the formation of reverse micelles upon introduction of $\mathrm{AgNO}_{3}$ solution into a mixture of cyclohexane and Igepal CO-520 (Figure 1). The hydrophilic polyethylene oxide chains of the surfactant then partially reduce the silver cations, ${ }^{[19,20]}$ a process which is completed by an additional reducing agent. In a next step, the silica precursors APTS and TEOS are introduced into the micellar system. The APTS ethanolic solution is soluble in the water phase, hence is located inside the micelle and is expected to coordinate to silver via its $\mathrm{N}$-donor atoms, keeping the metal particles thus inside the micelle. On the other hand, since TEOS is practically insoluble in water, it remains in the oil phase ${ }^{[18]}$ and hydrolyzes slowly on the boundary phase of the micelle. After injection of aqueous ammonia-a catalyst for the hydrolysis of silica precursors ${ }^{[14,15,18]}$ — the formed droplets are prone to collide with already existing micelles. We have observed that relatively longtime intervals between the addition of the individual ingredients are necessary to provide their uniform distribution and thus form the basis of product homogeneity. Hydrolysis and polycondensation of silica precursors under basic conditions lead then to the formation of a silica shell around the AgNPs. A high concentration of hydrazine (compared with the initial concentration of $\mathrm{AgNO}_{3}$ ) is required to preserve etching of the AgNPs by ammonia. The purification process with warm ultrapure water leads to the removal of unreacted APTS and subsequently to the formation of hollow porous silica nanocontainers. 


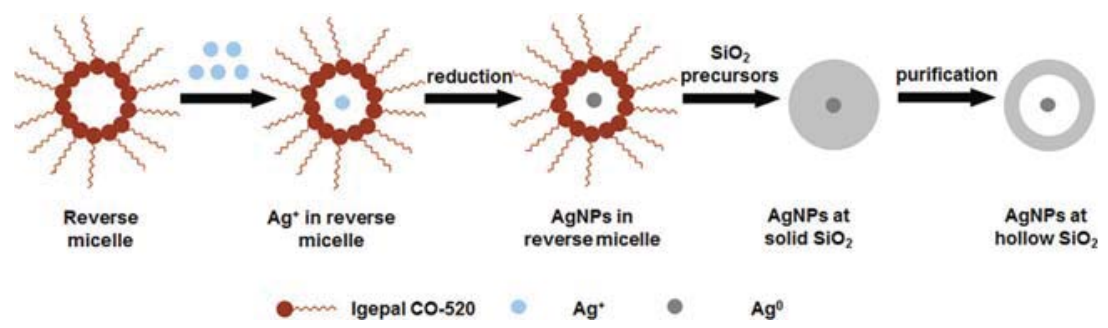

Figure 1. Schematic illustration for the formation of $\mathrm{Ag} @ \mathrm{SiO}_{2}$ nanorattles through the microemulsion approach.

Size and shape of AgNPs as well as the loading of silica with the AgNPs can be tuned by variations in the experimental parameters, such as the type of surfactant, the reducing agent, the concentration of reducing agent and silver nitrate, factors which are discussed in the following.

To obtain a nanorattle pattern for the incorporation of AgNPs into silica nanocontainers, the following requirements should be fulfilled: (a) AgNPs should be completely covered by a silica shell, i.e., should be large enough not to leak out through the silica pores, (b) silica hollow spheres should be homogenously loaded with silver, (c) no bulky silver should be formed outside the nanocontainers, and (d) a void between the silica shell and the AgNPs should be maintained.

It was reported that hydrophilic polyethylene oxide groups of nonionic surfactants feature properties to reduce $\mathrm{Ag}^{+}$to $\mathrm{Ag}^{0}$ and form hydroperoxide. In this case, the surfactant has a dual role, i.e., as a stabilizing and as a reducing agent. ${ }^{[19,20]}$ In our preliminary study (Paragraph S1, Supporting Information), we modified the procedure proposed by Viger and co-workers ${ }^{[21]}$ in order to prepare AgNPs covered by a silica shell. Two different nonionic surfactants, namely Triton X-100 and Igepal CO-520, were used in parallel experiments (Figure 2a,b). The main difference between them is the ratio of hydrophilic to hydrophobic parts (hydrophiliclipophilic balance, $\mathrm{HLB}_{\text {Triton X-100 }}=13.6,{ }^{[22]}$ $\operatorname{HLB}_{\text {Igepal CO-520 }}=10^{[12]}$ ) and the branching (complexity) of the hydrophobic chain. As the latter does not contribute to the reduction of $\mathrm{Ag}^{+}$, we will focus our further considerations on the influence of the hydrophilic chains. For commercially available Triton X-100, the number of ethoxy groups varies between 9 and 10, whereas for Igepal CO-520, it is 5, meaning that the former has more reducing sites and should offer better reducing properties than the latter. However, on addition of $\mathrm{AgNO}_{3}$ solution, the yellow color indicating the formation of $\mathrm{AgNPS}^{[8,19,20,23-25]}$ occurred only in the case of the microemulsion with Igepal CO-520, but not with Triton X-100. Completion of reduction initiated by addition of hydrazine caused a change of the Triton $\mathrm{X}$-100-containing microemulsion from transparent to black, suggesting the presence of bulky metallic silver, whereas the color of the
Igepal CO-520 mixture remained unchanged. Subsequent introduction and hydrolysis of TEOS aimed at covering the formed AgNPs in order to enhance their stability. UV-vis spectra of the purified samples showed absorptions at $\lambda_{\max }=400$ and $415 \mathrm{~nm}$ for samples prepared in Igepal CO-520 and Triton X-100, respectively (Figure S1, Supporting Information). The reduction of $\mathrm{Ag}^{+}$in a microemulsion consisting of Igepal CO-520 yielded the formation of a homogenous sample with spherical AgNPs of narrow size between 4 and $10 \mathrm{~nm}$ inside a spherical silica shell (13-20 $\mathrm{nm}$ in diameter), and some uncoated larger AgNPs of $20-30 \mathrm{~nm}$ in size (Figure $2 \mathrm{~b}$ ). The samples prepared in Triton X-100 provided AgNPs with a broad size distribution and were not or not completely coated with silica (Figure 2a).

Liz-Marzán and Lado-Touriño ${ }^{[20]}$ suggested an influence of the geometry of surfactants on the formation of AgNPs.
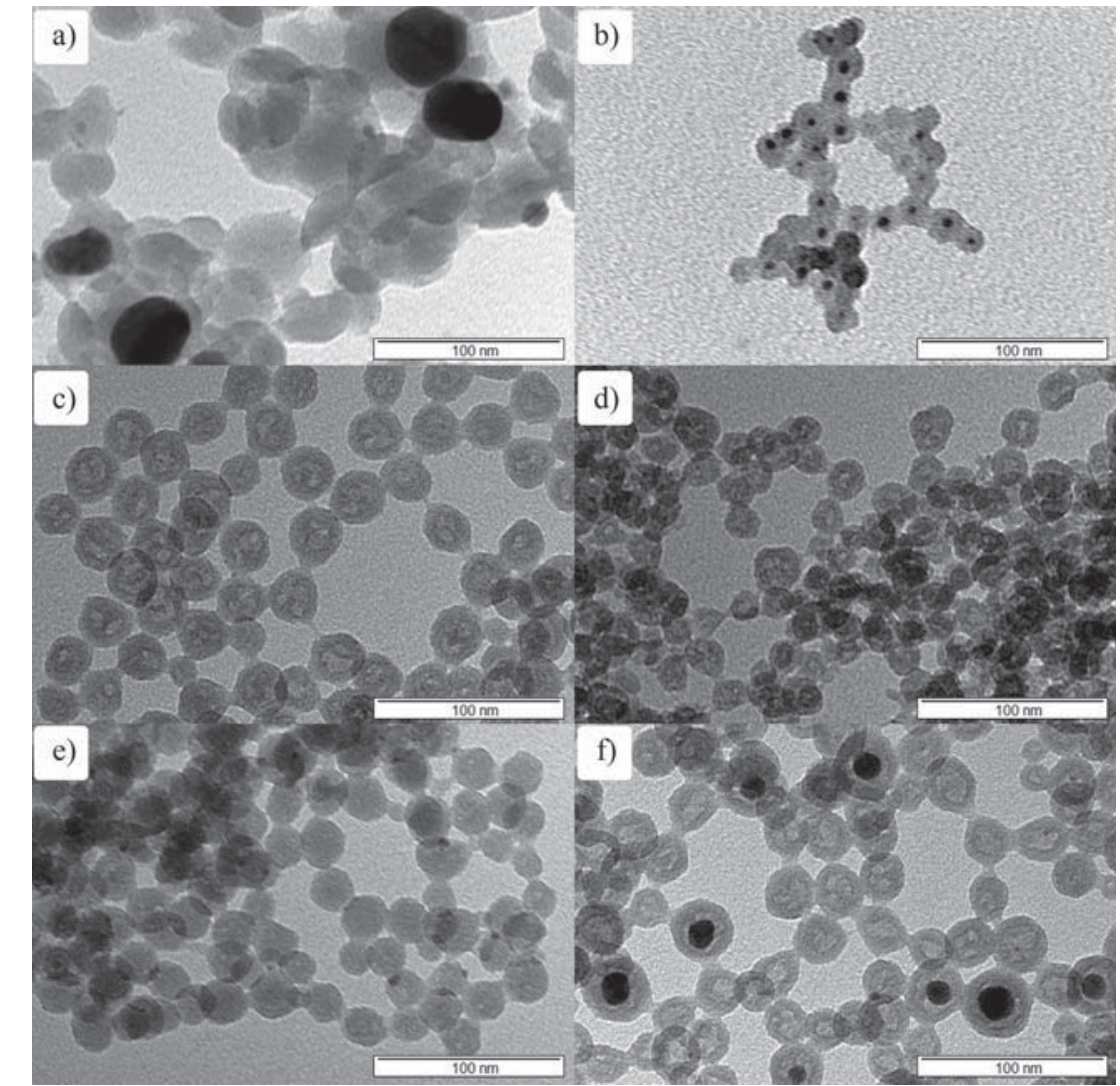

Figure 2. TEM images of $\mathrm{Ag} @ \mathrm{SiO}_{2}$, prepared using a) Triton X-100, b) Igepal CO-520 as surfactant; c) citric acid, d) ascorbic acid, e) sodium borohydride, f) hydrazine as reducing agent. Scale bar: $100 \mathrm{~nm}$. 
Although these differences do not occur between Triton X-100 and Igepal CO-520, it should be noticed that hydrophilic ethoxy groups point into the interior of the micelle and their interactions are limited by the size of the water pool. This leads us to the conclusion that although both surfactants are nonionic and have a similar structure, the length of hydrophilic chains has a crucial role in the formation of homogenously sized AgNPs in the microemulsion.

AgNPs are known to be prone to etching by ammonia, ${ }^{[26,27]}$ the presence of which, however, is necessary to initiate the hydrolysis of silica precursors. This challenge can be dealt with by the proper choice of the reducing agent. Among both inorganic and organic reducing agents of different strengths, citric acid, ascorbic acid, sodium borohydride, and hydrazine were tested in our assays (Figure 2c-f). The two first agents, being organic acids, are expected to possess weaker reducing ability, whereas the other two should be stronger ones (Table S1, Supporting Information). After the introduction of $\mathrm{AgNO}_{3}$ into the microemulsion, the solutions turned orange, which indicates reduction. However, after $36 \mathrm{~h}$, the color disappeared in case of citric acid and weakened significantly in case of ascorbic acid as well as sodium borohydride. This might suggest that initially formed AgNPs were dissolved upon etching by ammonia. Only the solution containing hydrazine remained dark yellow, which indicates this reducing agent to be the most efficient one. Transmission electron microscopy (TEM) confirms indeed the formation of spherical AgNPs incorporated inside the silica shell (Figure 2f). It seems that even if the surface of the AgNPs is partially etched by ammonia, the large excess of hydrazine can
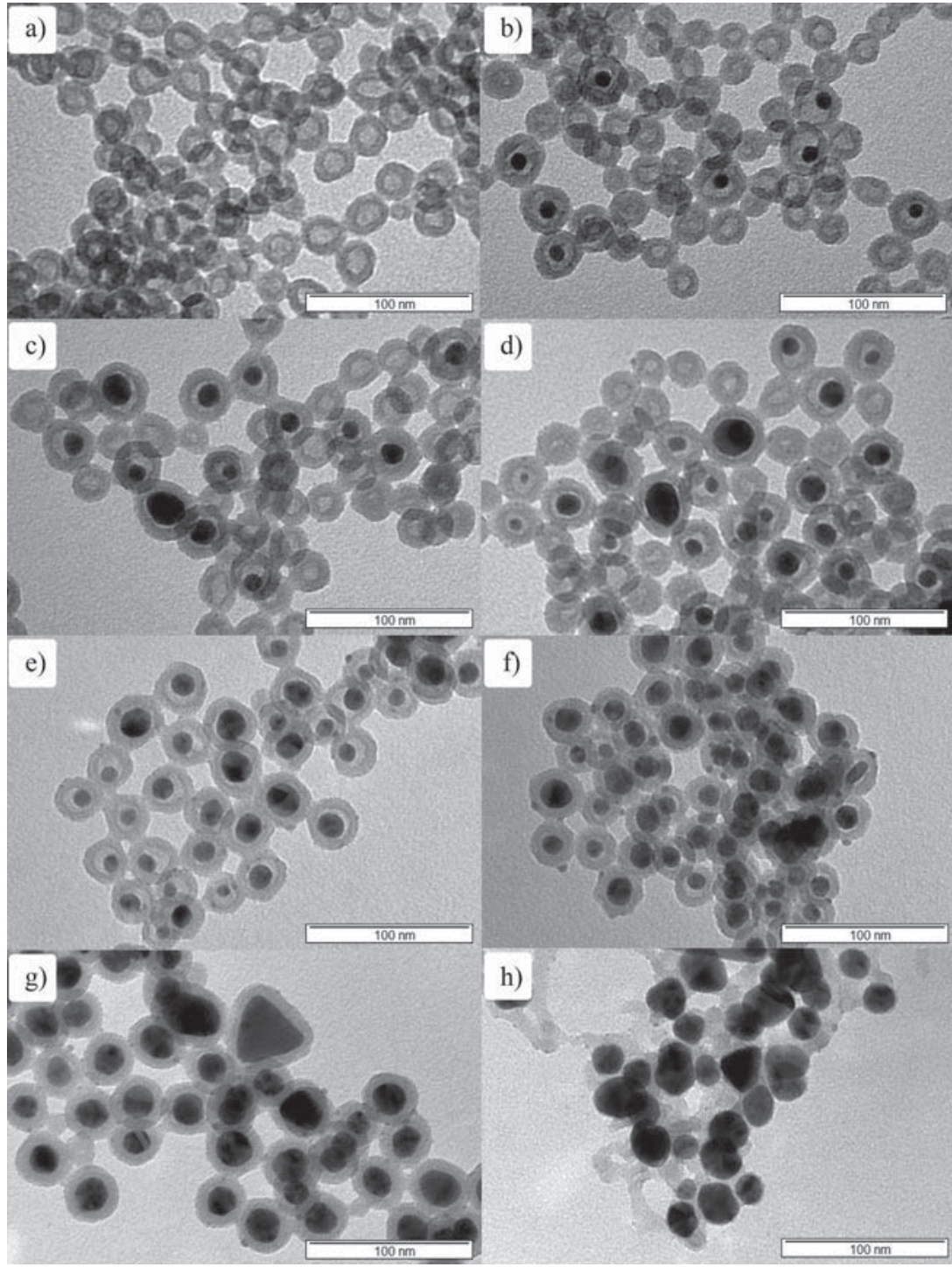

Figure 3. TEM images of a) $\mathrm{SiO}_{2}$ hollow spheres prepared using ultrapure water; $\mathrm{Ag@SiO}$ prepared using b) 0.01 , c) 0.05 , d) 0.1 , e) 0.2 , f) 0.3 , g) 0.5 , h) $1 \mathrm{M} \mathrm{AgNO}_{3}$. Scale bar: $100 \mathrm{~nm}$. prevent the escape of $\mathrm{Ag}^{+}$from the water pool of the micelle during the formation of the silica shell.

In standard preparation methods of AgNPs, a reducing agent is added to highly diluted (order of mu and lower) solutions of $\mathrm{Ag}^{+}$in the presence of stabilizing agents. ${ }^{[24,28,29]}$ On the other hand, using water-in-oil microemulsions, micelles act as nanoreactors ${ }^{[30]}$ - their cavity limits the size of NPs and therefore higher concentrations $(0.1 \mathrm{~N})$ of precursors can be used to obtain, for example, AgCl NPs. ${ }^{[31]}$

The micelles of our systems seem to provide sufficient separation for the formed AgNPs to prevent the formation of bulk silver. By increasing the concentration of the used $\mathrm{AgNO}_{3}$ solution (only ultrapure water, $0.01,0.05$, and $0.1 \mathrm{M}$ ), not only the size of the AgNPs increases but also the silica hollow spheres are loaded to a larger extent with AgNPs (Figure 3a-d) with a maximum yield of $35 \%$ of filled spheres $(n=1930$ particles counted). Interestingly, the shape of $\mathrm{SiO}_{2}$ largely depends on the size and the shape of the AgNPs. In case of small spherical
AgNPs, $\mathrm{SiO}_{2}$ nanocontainers preserve the spherical form and the void between the AgNPs and the silica shell is well visible (Figure 3b-d). However, in case of larger AgNPs of shapes other than spherical, the silica shell adopts their shape and covers the AgNPs. In addition, at higher concentrations of $\mathrm{AgNO}_{3}$, their shapes diverge from spherical to triangular and rod-like forms and the silica shell adjusts tightly around them with hardly any void visible (Figure S2, Supporting Information). As seen in Figure $3 \mathrm{e}-\mathrm{g}$, further increase of the $\mathrm{AgNO}_{3}$ concentration results in the higher loading of AgNPs, which at a concentration of $0.2 \mathrm{~m}$ reaches $100 \%$ (Figure $3 \mathrm{e}$ ). This concentration seems to impose the upper limit for the $\mathrm{AgNO}_{3}$ concentration in our system in order to obtain the nanorattle pattern. Indeed, the void between the AgNPs and the silica wall is hardly preserved at a concentration of $0.3 \mathrm{M}$ (Figure $3 \mathrm{f}$ ) and completely vanishes using $0.5 \mathrm{M} \mathrm{AgNO}_{3}$ solution, resulting in tight coating i. e. core/ shell $\mathrm{Ag} @ \mathrm{SiO}_{2}$ (Figure 3g). A concentration of the $\mathrm{AgNO}_{3}$ 


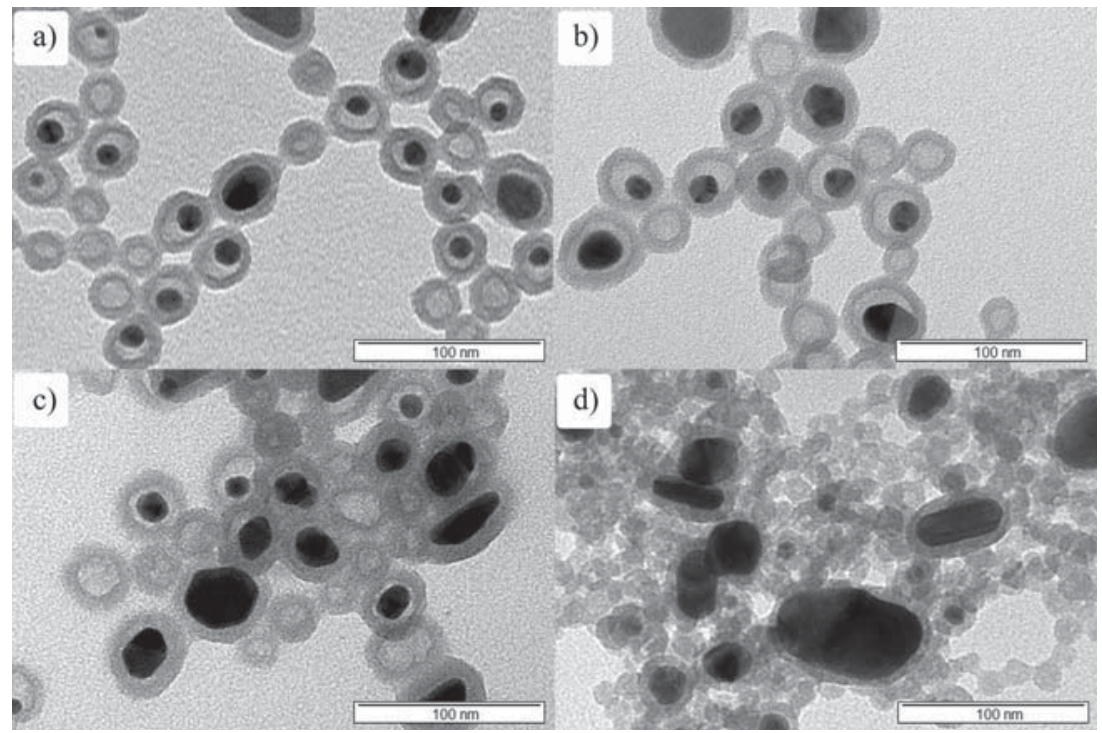

Figure 4. TEM images of $\mathrm{Ag} @ \mathrm{SiO}_{2}$, prepared using a) 20, b) 10, c) 5, d) 1 m hydrazine. Scale bar: $100 \mathrm{~nm}$.

solution of $1 \mathrm{~m}$ leads to the formation of larger AgNPs, which are not encapsulated in nanocontainers. In addition, silica does not form any more spherical shapes (Figure 3h). Worth noticing is the fact that this concentration does not exclude formation of $\mathrm{Ag} @ \mathrm{SiO}_{2}$ structures of tight coatings. Indeed, this can be achieved by increasing the hydrazine content used for the reduction of silver cations from 50 to $250 \mu \mathrm{L}$. In this case, small AgNPs are obtained, which are completely covered with a thin silica layer (Figure S3, Supporting Information).

Various other NPs, such as $\mathrm{Cu},{ }^{[32]} \mathrm{Au},{ }^{[33]} \mathrm{Pd}^{[34]}$ or $\mathrm{Ni}^{[35,36]}$ have also been prepared by reduction in systems stabilized by surfactants. The observed general trend is the following: at constant concentration of metal precursor, the NP size decreases with the increasing concentration of hydrazine. ${ }^{[32-36]}$ The correlation between size and spectroscopic properties demonstrated that for the larger NPs, the maximum of absorption is more shifted to the longer wavelengths. ${ }^{[24,37,38]}$ We observed aforementioned effects while maintaining the same concentration of $\mathrm{AgNO}_{3}(0.1 \mathrm{M})$ and varying the concentration of hydrazine (20, $10,5$, and $1 \mathrm{M})$. At relatively high concentration of hydrazine, 20 and $10 \mathrm{~m}$, the maximum of absorption is at 403 and $404 \mathrm{~nm}$, respectively. However, at lower concentrations, 5 and $1 \mathrm{~m}$, the absorbance maxima are shifted toward longer wavelengths and occur at $\lambda_{\max }=408$ and $422 \mathrm{~nm}$, respectively (Figure S4, Supporting Information). Since these wavelengths correspond to the size differences of AgNPs, the shifts of maxima in the UVvis spectra indicate the increase of AgNPs size, as confirmed by TEM (Figure 4a-d). In addition, with the lowest concentration of hydrazine, no hollow silica nanocontainers were observed (Figure $4 \mathrm{~d}$ ) and the size of solid silica spheres was significantly smaller than the size of hollow $\mathrm{SiO}_{2}$ or hollow $\mathrm{SiO}_{2}$ filled with small AgNPs. Altogether, this leads us to the conclusion that the lower the used concentration of hydrazine, the larger the size of AgNPs and the better they are protected from etching. Hence, hydrazine can be also considered as an AgNP protecting/capping agent.
AgNPs are also known to possess catalytic properties, ${ }^{[8,9]}$ for example, for the reduction of dyes, such as methylene blue, by sodium borohydride $\left(\mathrm{NaBH}_{4}\right){ }^{[9]}$ We therefore wanted to test if our Ag@ $\mathrm{SiO}_{2}$ nanorattles are active catalysts. At low concentrations of $\mathrm{NaBH}_{4}$, a reduction of methylene blue does not or only weakly occurs (Figure S5, Supporting Information). However, addition of $\mathrm{NaBH}_{4}$ to the dye in presence of our $\mathrm{Ag} @ \mathrm{SiO}_{2}$ accelerates the reduction and results in vanishing of the blue color of the dye (Figure 5; Figure S6, Supporting Information). Since our AgNPs are inside the $\mathrm{SiO}_{2}$ nanocontainers, the reaction is slower as compared with surfaceexposed AgNPs ${ }^{[9]}$ as diffusion in and out of the nanocontainers has to occur. Much like Wu et al., ${ }^{[15]}$ we observed that the smaller the size of the NPs, the higher their catalytic activities (Figure $5 d$ ). In agreement with the study of Jiang and co-workers, ${ }^{[9]}$ the higher the concentration of AgNPs, the faster the reduction of methylene blue (Figure $5 \mathrm{a}-\mathrm{c}$ ).

It is well known that the presence of pores in the wall enables transport of molecules inside and outside the nanocontainers. ${ }^{[39,40]}$ Therefore, influencing the speed at which reactants are transported though the wall should have an impact on the catalytic properties as well. The porosity of empty $\mathrm{SiO}_{2}$ (Figure 3a) was investigated using nitrogen adsorption-desorption measurements. As shown in Figure 6a, the nanocontainers exhibit a type-IV isotherm with a large hysteresis loop at a relative pressure between 0.7 and $1.0 \mathrm{P} / \mathrm{P}_{0}$. Those nanocontainers demonstrated a relatively large surface area $\left(160 \mathrm{~m}^{2} \mathrm{~g}^{-1}\right)$ and pore volume $\left(1.14 \mathrm{~cm}^{3} \mathrm{~g}^{-1}\right)$, which is in agreement with previously reported $\mathrm{SiO}_{2}$ nanocontainers prepared using the microemulsion technique $\left(164 \mathrm{~m}^{2} \mathrm{~g}^{-1}\right.$ and $0.79 \mathrm{~cm}^{3} \mathrm{~g}^{-1}$, respectively). ${ }^{[15]}$ Those differences in the pore volumes are rather not related to the slight differences in diameters and wall thicknesses of those nanocontainers $(26.2 \pm 2.6 \text { and } 6 \mathrm{~nm} \text {, respectively })^{[15]}$ and ours $(25.3 \pm 2.5$ and $5.7 \pm 1.4 \mathrm{~nm}$, respectively). The pore diameter was determined to be approximately $28.5 \mathrm{~nm}$ (Figure 6b), which is attributed to the interparticle voids. Since the pores are too miniscule to be detected via $\mathrm{N}_{2}$ adsorption-desorption measurements, we performed a simple test to prove their presence. A sample of $\mathrm{Ag} @ \mathrm{SiO}_{2}$ nanorattles (Figure 6c) was treated with 65\% nitric acid, which leads to dissolution of AgNPs and thus occurrence of empty silica nanocontainers with preserved shapes (Figure 6d). This evidently proves presence of pores in the wall, which can act as pathways for molecule migration in and out of the nanocontainers.

Several studies reported the preparation of nanorattles with tunable shell thickness. ${ }^{[1,42]}$ However, to best authors' knowledge, no research was done to tune the wall thickness using the microemulsion approach. As APTS has the role of void determining agent, we assumed that changing the concentration of TEOS should enable the control of the shell thickness. Indeed, using increasing quantities of TEOS: 50, 100, 200, and 

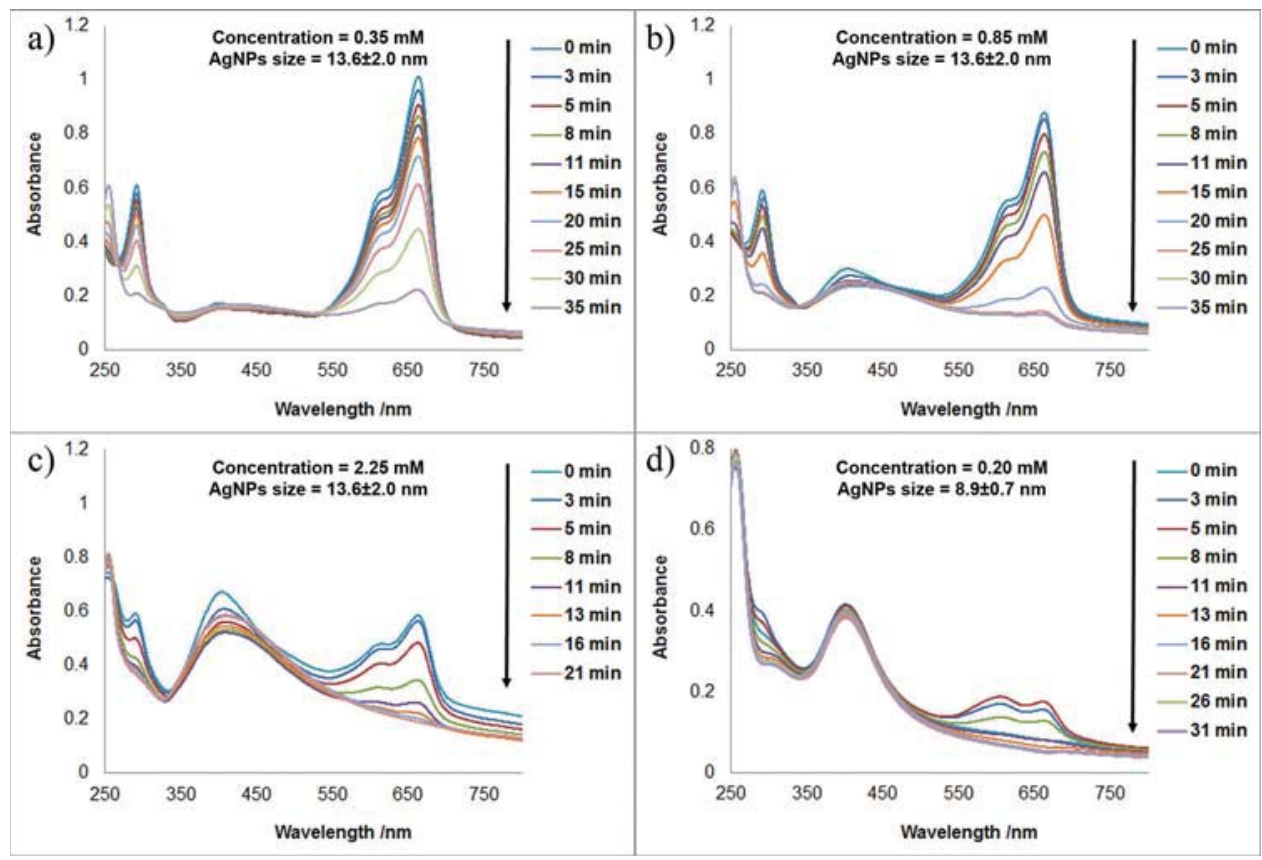

Figure 5. Time-resolved UV-Vis spectra of methylene blue after addition of $\mathrm{Ag} @ \mathrm{SiO}_{2}$ (Figure 3d, AgNPs size = $13.6 \pm 2 \mathrm{~nm}$ ) suspensions at different concentrations of silver a) $0.35 \times 10^{-3} \mathrm{M}$, b) $0.85 \times 10^{-3} \mathrm{M}$, c) $2.25 \times 10^{-3} \mathrm{M}$, and d) $\mathrm{Ag} @ \mathrm{SiO}_{2}$ (Figure 3b, AgNPs size $=8.9 \pm 0.7 \mathrm{~nm}$ ) suspension at concentrations of silver $0.20 \times 10^{-3} \mathrm{M} .8 \times 10^{-3} \mathrm{M} \mathrm{NaBH}_{4}$ solution was used for the reduction.

$300 \mu \mathrm{L}$, the wall can be tuned to $5.3 \pm 1.1 \mathrm{~nm}(n=279), 7.0 \pm$ $1.2 \mathrm{~nm}(n=309), 9.2 \pm 1.4 \mathrm{~nm}(n=198)$, and $10.3 \pm 1.6 \mathrm{~nm}$ $(n=271)$, respectively. As shown in Figure 7, varying the concentration of TEOS not only influences the wall thickness but
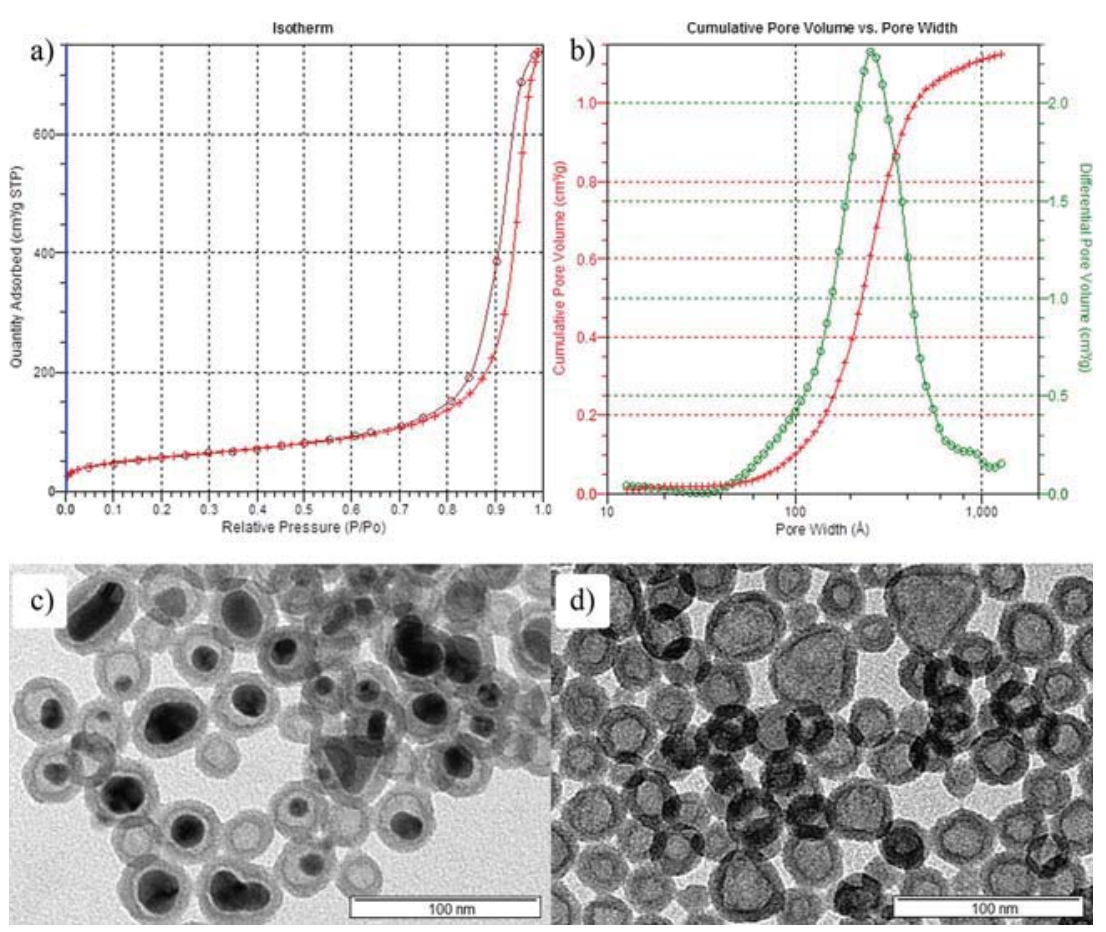

Figure 6. a) Nitrogen absorption-desorption isotherm, b) the pore size distribution, TEM of c) $\mathrm{Ag} @ \mathrm{SiO}_{2}$ nanorattles with irregular shape, and d) $\mathrm{SiO}_{2}$ nanocontainers with irregular shape obtained by removing AgNPs. also affects the loading with AgNPs, leading to the formation of AgNPs outside the $\mathrm{SiO}_{2}$ nanocontainers (Figure 7a,c,d). Those NPs are closely associated with the walls most probably due to the presence of amino groups. This is in agreement with a study of Wang et al. ${ }^{[43]}$ where PdNPs were chemisorbed on the surface of silica functionalized with APTS.

In conclusion, we have successfully synthetized $\mathrm{Ag} @ \mathrm{SiO}_{2}$ nanorattles using a onepot synthesis via encapsulation in a water-inoil microemulsion. After screening a variety of conditions, it can be concluded that nanorattles of desired properties can be tuned as a function of the circumstances: (a) the use of Igepal CO-520 as surfactant provides the formation of homogenous AgNPs, uniformly covered by a silica shell; (b) increasing the concentration of $\mathrm{AgNO}_{3}$ in the water pool of the micelles leads to the formation of larger and more shape-diverse AgNPs; (c) hydrazine, as a strong reducing agent, not only leads to well-defined AgNPs but also protects them from the etching properties of aqueous ammonia; and (d) the wall thickness can be controlled by varying the concentration of TEOS. Those one-pot reactions enable the preparation of empty silica nanocontainers and $\mathrm{Ag} @ \mathrm{SiO}_{2}$ yolk/shell and core/shell NPs. Furthermore, we have shown that $\mathrm{Ag} @ \mathrm{SiO}_{2}$ nanorattles exhibit good catalytic properties in the reduction of methylene blue by $\mathrm{NaBH}_{4}$. 


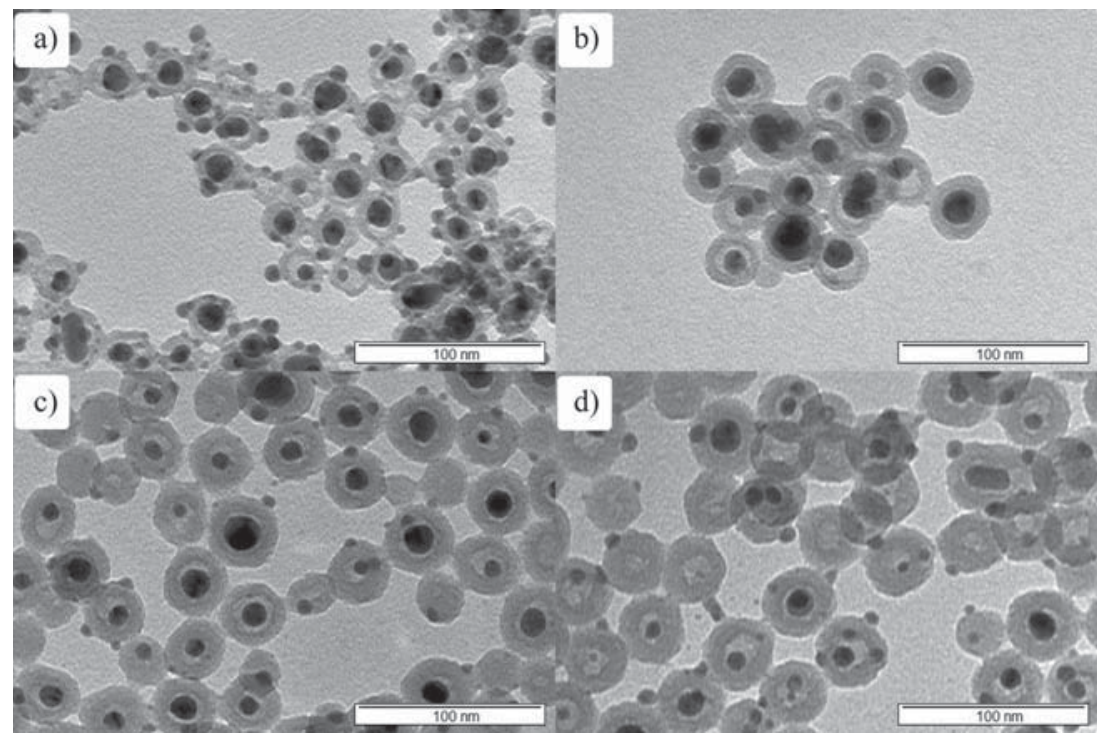

Figure 7. TEM images of $\mathrm{Ag} @ \mathrm{SiO}_{2}$, prepared using a) 100, b) 200, c) 300, d) $400 \mu \mathrm{L}$ of TEOS. Scale bar: $100 \mathrm{~nm}$.

\section{Experimental Section}

Synthesis of Ag@SiO by $\mathrm{Wu}$ and co-workers[15] for silica hollow spheres and work by Viger and co-workers ${ }^{[2]}$ for the preparation of AgNPs inside solid silica in microemulsion. After cyclohexane $(29.6 \mathrm{~g})$ was mixed with Igepal CO-520 $(3.5 \mathrm{~mL})$, the aqueous solution $(1.4 \mathrm{~mL})$ was added dropwise and the microemulsion was vigorously stirred over $1-2 \mathrm{~h}$ to equilibrate. Then, the reducing agent was introduced, after another $2 \mathrm{~h}$, TEOS $(200 \mu \mathrm{L})$, and $12.5 \mathrm{v} / \mathrm{v} \%$ ethanolic solution of APTS $(50 \mu \mathrm{L})$ were added and left for another $2 \mathrm{~h}$. Addition of $\mathrm{NH}_{4} \mathrm{OH}(500 \mu \mathrm{L})$ initiated hydrolysis and polycondensation of silica precursors. After $36 \mathrm{~h}$, the microemulsion was destabilized by addition of $\mathrm{EtOH}(25 \mathrm{~mL})$ and followed by a purification process, which involved centrifugation (15 $000 \mathrm{rpm}, 30 \mathrm{~min}, \mathrm{rt}$ ), twice washing with $\mathrm{EtOH}$, twice washing with ultrapure water $(15000 \mathrm{rpm}$, $15 \mathrm{~min}, \mathrm{rt})$, and washing with warm ultrapure water $\left(50^{\circ} \mathrm{C}, 40 \mathrm{~min}\right.$, afterward centrifugation $15000 \mathrm{rpm}, 15 \mathrm{~min}, \mathrm{rt}$ ). Each washing step involved ultrasonication in order to disperse the nanoparticles and provide equal washing. Samples were dried under high vacuum. Detailed information about the experimental procedure can be found in the Supporting Information (Table S2).

Characterization: The morphology of the samples was characterized by transmission electron microscopy (TEM) using a FEl/Philips CM-100 Biotwin transmission electron microscope (the operating voltage $=80 \mathrm{kV}$, in bright-field mode). After hot ultrapure washing, a drop of diluted and sonicated suspension was deposited on the TEM grid (Electron Microscopy Sciences, CF 300-Cu, Carbon Film on 300 Square Mesh Copper Grids), covered in order to avoid contamination by dust from air and let dry overnight. UV-Vis spectrometry (UV-Vis spectrometer, Lambda40, Perkin Elmer) of a suspension after hot ultrapure wash was done within a wavelength range from 250 to $800 \mathrm{~nm}$. Analysis whether dried samples exhibit crystalline structure was done using X-ray powder diffractometry (The STADI P, STOE Transmission X-ray Powder Diffraction System; copper source, $2 \theta$ from 5-90, duration time: 2 h). Nitrogen adsorption-desorption isotherms were performed on an Accelerated Surface Area and Porosimetry System (Micromeritics, ASAP 2010) at $-195.8^{\circ} \mathrm{C}$ using liquid nitrogen. $\mathrm{SiO}_{2}$ nanocontainers were degassed at $130^{\circ} \mathrm{C}$ over $20 \mathrm{~h}$ under pressure of $3 \mu \mathrm{m} \mathrm{Hg}$. Calculations were performed using the MicroActive programme. The surface area (correlation coefficient $=0.9993$ ) as well as the pore size were obtained by the Brunauer-Emmett-Teller method.
Determination of Catalytic Properties: Typically, a few milligrams of Ag@ $\mathrm{SiO}_{2}$ were dispersed in $1 \mathrm{~mL}$ of ultrapure water, sonicated, and let to sediment. $200 \mu \mathrm{L}$ of $\mathrm{Ag} @ \mathrm{SiO}_{2}$ suspension was mixed with methylene blue $\left(2 \mathrm{~mL}, 2 \times 10^{-5} \mathrm{~mol} \mathrm{~L}^{-1}\right)$. After addition of $200 \mu \mathrm{L}$ of $8 \times 10^{-3} \mathrm{M} \mathrm{NaBH}_{4}$ solution, time-resolved UV-vis spectroscopy was carried out within a wavelength range from 250 to $800 \mathrm{~nm}$. After the UV-vis measurement, the suspension was sonicated and vortexed, then $2 \mathrm{~mL}$ was mixed with $65 \%$ nitric acid $(800 \mu \mathrm{L})$ and ultrapure water $(2.2 \mathrm{~mL}$ ). After filtration (pore size $=0.22 \mu \mathrm{m}$ ), the initial concentration of silver in the $\mathrm{Ag} @ \mathrm{SiO}_{2}$ suspension was determined by inductively coupled plasma optical emission spectrometry (ICP-OES) using PerkinElmer Optical Emission Spectrometer (Optima 7000 DV).

\section{Supporting Information}

Supporting Information is available

\section{Acknowledgements}

The authors thank the University of Fribourg, the Fribourg Center for Nanomaterials FriMat, and the Swiss National Science Foundation for the generous funding of the project. This project was carried out within The National Research Programme NRP-62 "Smart materials." The authors thank Dr. Aurélien Crochet, the Fribourg Center for Nanomaterials for his kind help with surface area and porosimetry analysis.

[1] R. G. Chaudhuri, S. Paria, Chem. Rev. 2012, 112, 2373

[2] J. Liu, S. Z. Qiao, J. S. Chen, X. W. (D.) Lou, X. Xing, G. Q. (M.) Lu, Chem. Commun. 2011, 47, 12578.

[3] S. Kalele, S. W. Gosavi, J. Urban, S. K. Kulkarni, Curr. Sci. 2006, 91, 1038.

[4] W. Zhao, H. Chen, Y. Li, L. Li, M. Lang, J. Shi, Adv. Funct. Mater. 2008, 18, 2780.

[5] L. Wang, J. Shi, Y. Zhu, Q. He, H. Xing, J. Zhou, F. Chen, Y. Chen, Langmuir 2012, 28, 4920.

[6] H. Yin, Z. Ma, M. Chi, S. Dai, Catal. Today 2011, 160, 87.

[7] L. H. Yao, Y. X. Li, J. Zhao, W. J. Ji, C. T. Au, Catal. Today 2010, 158, 401.

[8] T. Ung, L. M. Liz-Marzán, P. Mulvaney, J. Phys. Chem. B 1999, 103, 6770.

[9] Z.-J. Jiang, C.-Y. Liu, L.-W. Sun, J. Phys. Chem. B 2005, 109, 1730.

[10] Y. Chen, C. Wang, H. Liu, J. Qiu, X. Bao, Chem. Commun. 2005, 5298.

[11] M. Jafelicci Jr., M. R. Davolos, F. J. dos Santos, S. J. de Andrade, J. Non-Cryst. Solids 1999, 247, 98.

[12] F. J. Arriagada, K. Osseo-Asare, J. Colloid Interface Sci. 1999, 211, 210.

[13] C.-L. Chang, H. S. Fogler, Langmuir 1997, 13, 3295

[14] Y.-S. Lin, S.-H. Wu, C.-T. Tseng, Y. Hung, C. Chang, C.-Y. Mou, Chem. Commun. 2009, 3542. 
[15] S.-H. Wu, C.-T. Theng, Y.-S. Lin, C.-H. Lin, Y. Hung, C.-Y. Mou, J. Mater. Chem. 2011, 21, 789.

[16] T. Li, J. Moon, A. A. Morrone, J. J. Mecholsky, D. R. Talham, J. H. Adair, Langmuir 1999, 15, 4328.

[17] W. Wang, Z. Li, B. Gu, Z. Zhang, H. Xu, ACS Nano 2009, 3, 3493.

[18] S.-H. Chung, D.-W. Lee, M.-S. Kim, K.-Y. Lee, J. Colloid Interface Sci. 2011, 355, 70.

[19] M.-H. Lee, S.-G. Oh, K.-D. Suh, D.-G. Kim, D. Sohn, Colloids Surf. A: Physicochem. Eng. Aspects 2002, 210, 49.

[20] L. M. Liz-Marzán, I. Lado-Touriño, Langmuir 1996, 12, 3585.

[21] M. L. Viger, L. S. Live, O. D. Therrien, D. Boudreau, Plasmonics 2008, 3, 33.

[22] X. Jin, D. A. Streett, C. A. Dunlap, M. E. Lyn, Biol. Control 2008, 46, 226.

[23] A. Henglein, Chem. Mater. 1998, 10, 444.

[24] R. Jin, Y. W. Cao, C. A. Mirkin, K. L. Kelly, G. C. Schatz, J. G. Zhang, Science 2001, 294, 1901.

[25] J.-M. Lee, D.-W. Kim, T.-H. Kim, S.-G. Oh, Mater. Lett. 2007, 61, 1558.

[26] C. Xue, X. Chen, S. J. Hurst, C. A. Mirkin, Adv. Mater. 2007, 19, 4071.

[27] Y. Yin, Y. Lu, Y. Sun, Y. Xia, Nano Lett. 2002, 2, 427.

[28] Y. Sun, B. Mayers, Y. Xia, Nano Lett. 2003, 3, 675.
[29] A. Callegari, D. Tonti, M. Chergui, Nano Lett. 2003, 3, 1565.

[30] V. Pillai, P. Kumar, M. J. Hou, P. Ayyub, D. O. Shah, Adv. Colloid Interface Sci. 1995, 55, 241.

[31] R. P. Bagwe, K. C. Khilar, Langmuir 1997, 13, 6432.

[32] S.-H. Wu, D.-H. Chen, J. Colloid Interface Sci. 2004, 273, 165.

[33] C. L. Chiang, J. Colloid Interface Sci. 2001, 239, 334.

[34] C.-C. Wang, D.-H. Chen, T.-C. Huang, Colloids Surf. A: Physicochem. Eng. Aspects 2001, 189, 145.

[35] D.-H. Chen, C.-H. Hsieh, J. Mater. Chem. 2002, 12, 2412.

[36] D.-H. Chen, S.-H. Wu, Chem. Mater. 2000, 12, 1354.

[37] S. Link, M. A. El-Sayed, J. Phys. Chem. B 1999, 103, 8410.

[38] D. L. Van Hyning, C. F. Zukoski, Langmuir 1998, 14, 7034.

[39] Y. Yang, X. Liu, X. Li, J. Zhao, S. Bai, Q. Yang, Angew. Chem. 2012, $124,9298$.

[40] X. Kang, Z. Cheng, D. Yang, P. Ma, M. Shang, C. Peng, Y. Dai, J. Lin, Adv. Funct. Mater. 2012, 22, 1470.

[41] B. Liu, J. Wang, S. Sun, X. Wang, M. Zhao, W. Zhang, H. Zhang, X. Yang, RSC Adv. 2013, 3, 18506.

[42] G. Song, C. Li, J. Hu, R. Zou, K. Xu, L. Han, Q. Wang, J. Yang, Z. Chen, Z. Qin, K. Ruan, R. Hu, J. Mater. Chem. 2012, 22, 17011.

[43] Y. Wang, A. V. Biradar, C. T. Duncan, T. Asefa, J. Mater. Chem. 2010, 20, 7834. 